of blood in RA were found significantly more often than in OA (respectively $14.4 \%$ and $5.7 \%$ of cases; $P<0.001$ ). The number of cases requiring cancellation of anticoagulant therapy in patients with RA was significantly higher compared with the OA group $(6.6 \%$ and $1.4 \%$, respectively). Slow wound healing in RA was more common ( $n=56 ; 26.4 \%$ ) than in $\mathrm{OA}(\mathrm{n}=14 ; 5.1 \%)$. In patients who underwent monotherapy with calcium nadroparin VTE occurred more often than when using combination therapy $(p<0.0001)$ and more often than in the group of dabigatran etexilate $(p=0.054)$.

Conclusion: The frequency of VTE, the risk of bleeding and complications of postoperative wound in patients with RA and OA after THA were analyzed. So, our study determined the dependence of VTE complications and bleeding risk according to the patient's underlying nosology. Also the advantage of combined postoperative therapy over others was evaluated. Disclosure of Interests: None declared

DOI: 10.1136/annrheumdis-2019-eular.6996

\section{AB1289 TESTING DIFFERENT ITEMS INCLUDED IN THE DEFINITION OF REMISSION IN A MULTICENTRE SLE COHORT}

Francesca Saccon ${ }^{1}$, Margherita Zen ${ }^{1}$, Mariele Gatto ${ }^{1}$, Domenico Pe Margiotta ${ }^{2}$, Fulvia Ceccarelli $^{3}$, Giulia Frontini ${ }^{4}$, Gabriella Moroni ${ }^{4}$, Alessandra Bortoluzzi ${ }^{5}$, Marcello Govoni ${ }^{5}$, Viola Signorini ${ }^{6}$, Marta Mosca ${ }^{6}$, Francesca Dall'ara ${ }^{7}$, Angela Tincani ${ }^{7}$, Anna Chiara Frigo ${ }^{8}$, Antonella Afeltra ${ }^{2}$, Fabrizio Conti ${ }^{3}$, Andrea Doria'. ' 'University of Padova, Division of Rheumatology, Department of Medicine DIMED, Padova, Italy; ${ }^{2}$ Università Campus Bio-Medico di Roma, Unit of Allergy, Clinical immunology and Rheumatology, Roma, Italy, ${ }^{3}$ Sapienza University of Roma, Dipartimento di Medicina Interna e Specialità Mediche, Roma, Italy; ${ }^{4}$ Fondazione Ca' Granda IRCCS Ospedale Maggiore Policlinico Milano, Division of Nephrology, Milano, Italy, ${ }^{5}$ University of Ferrara and Azienda OspedalieroUniversitaria S. Anna, Dep. of Medical Sciences, Ferrara, Italy; ${ }^{6}$ University of Pisa, Rheumatology Unit, Dep. of Internal Medicine, Pisa, Italy, ${ }^{7}$ University of Brescia, Rheumatology and Clinical Immunology Unit Spedali Civili and Dep. of Clinical and Experimental Science, Brescia, Italy; ${ }^{8}$ University of Padova, Dep. of CardiacThoracic-Vascular Sciences and Public Health Biostatistics, Padova, Italy

Background: Remission is the most desirable target in the treatment systemic lupus erythematosus (SLE) however, a universally accepted definition of remission in SLE is still missing.

Objectives: To test the contribution of the different items included into the currently used definitions of remission in SLE.

Methods: We studied 646 Caucasian patients from a multicentre lupus cohort followed for at least 5-years: female 585 (90.6\%), mean age at baseline $40.59 \pm 12.14$ years, mean disease duration $9.18 \pm 6.86$ years. Disease activity was assessed by clinical SLE Disease Activity Index 2000 (CSLEDAI) and SELENA-SLEDAI physician global assessment (PGA), and damage by Systemic Lupus International Collaborating Clinics/ACR Damage Index (SDI). To test the performance of the different items included in the definitions of SLE remission [1, 2] we identify 7 subtypes of remission: (1) $P G A(0-3)<0.5$; (2) cSLEDAl $=0$; (3) prednisone (PDN) $\leq 5 \mathrm{mg} /$ day; (4) $P G A<0.5$ and $P D N \leq 5 m g / d a y ; ~(5) ~ c S L E D A l=0$ and $P G A<0.5$; (6) cSLEDAl $=0$ and $P D N \leq 5 \mathrm{mg} /$ day; (7) $c S L E D A l=0$ and $P D N \leq 5 \mathrm{mg} /$ day and $P G A<0.5$. The effect of remission on SDI was evaluated by Poisson regression analysis.

Results: The number of patients achieving remission according to the different definitions is show in Figure 1. The proportion of patients who maintained prolonged remission (5-consecutive years) was: $P G A<0.5$ 13.0\%; cSLEDAl=0 18.4\%; PDN $\leq 5 \mathrm{mg} /$ day $34.4 \%$; PGA $<0.5$ and $P D N \leq 5 \mathrm{mg} /$ day $12.5 \%$; cSLEDAl $=0$ and $P G A<0.512 .7 \%$; cSLEDAl $=0$ and $P D N \leq 5 \mathrm{mg} /$ day $16.6 \%$; cSLEDAl $=0$ and $P D N \leq 5 \mathrm{mg} /$ day and $P G A<0.512 .4 \%$.

Figure 1. Number of patients in remission according to the 3 single items

(prednisone $\leq 5 \mathrm{mg} /$ day; $\mathrm{PGA}<0.5 ; \mathrm{CSLEDAl}=0$ ) and their overlap during a follow-up period of 5 years for all the patients. A) Patients achieving at least 1-year remission. B) Patients achieving prolonged remission (5-consecutive years).
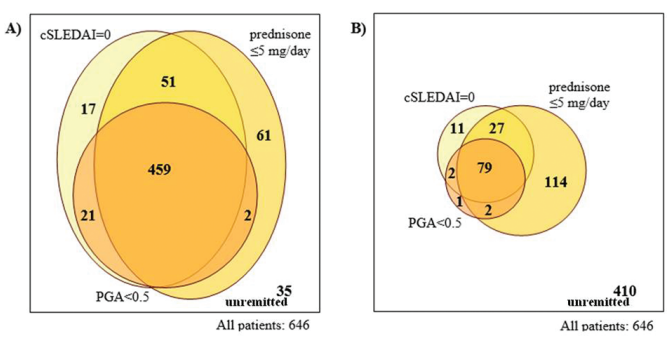

Table 1. Performance of 7 different remission subtypes in defining the risk of damage accrual (upper part) and the goodness-of-fit of the model (lower part).

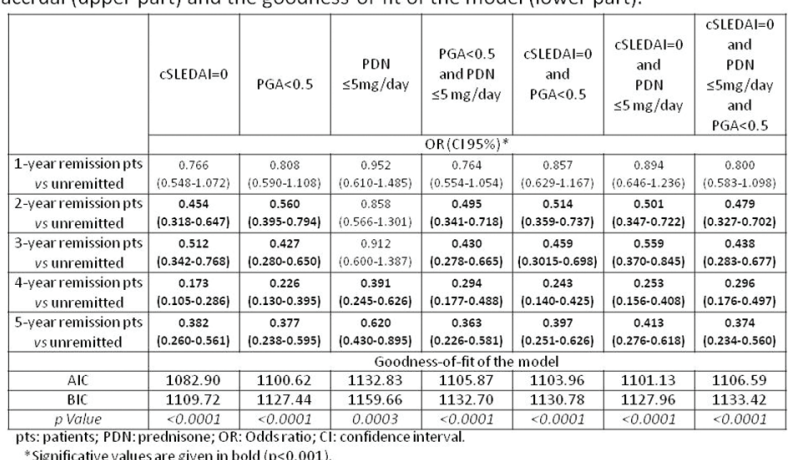

* Significative values are given in bold ( $\mathrm{p}<0.001$

Figure 1

When PGA $<0.5$ was added to CSLEDAI, $198(30.7 \%)$ patients lost 353 years in remission (1.8 years/patient): among them 195 (98.5\%) showed a $0.5 \leq P G A \leq 1$ suggesting a low disease activity state (LDA). When, $P G A<0.5$ was added to cSLEDAI $=0$ and $P D N \leq 5 \mathrm{mg} /$ day, $151(23.5 \%)$ patients lost 254 years in remission (1.7 years/patient): among them 149 $(98.0 \%)$ showed a $0.5 \leq P G A \leq 1$ suggesting again LDA. All remission subtypes were protective against damage $(p<0.001)$, however cSLEDAl=0 showed the best performance (lower AIC) (Table 1). At multivariate analysis all remission subtypes lasting $\geq 2$ consecutive years were protective against damage $(p<0.001)$, except $P D N \leq 5 \mathrm{mg} /$ day which was protective after 4 consecutive years.

Conclusion: The prevalence and extent of damage significantly decreased as the time spent in remission increased, irrespective of the remission subtype. The addition of $P G A<0.5$ to $c S L E D A l=0$ or to $c S L E D A l=0$ and $\mathrm{PDN} \leq 5 \mathrm{mg} /$ day results in a decrease in the time spent in remission without significant difference in damage accrual.

\section{REFERENCES}

[1] van Vollenhoven R, et al. 2014

[2] Zen M, et al. 2015

Disclosure of Interests: Francesca Saccon: None declared, Margherita Zen: None declared, Mariele Gatto: None declared, Domenico PE Margiotta: None declared, Fulvia Ceccarelli: None declared, Giulia Frontini: None declared, Gabriella Moroni: None declared, Alessandra Bortoluzzi: None declared, Marcello Govoni: None declared, Viola Signorini: None declared, Marta Mosca Paid instructor for: GlaxoSmithKline, Lilly, UCB, Francesca Dall'Ara: None declared, Angela Tincani Consultant for: UCB, Pfizer, Abbvie, BMS, Sanofi, Roche, GSK, AlphaSigma, Lilly, Jannsen, Cellgene, Novartis, Anna Chiara Frigo: None declared, Antonella Afeltra Grant/research support from: MSD, PFIZER, ABBVIE, ROCHE, UCB Speakers bureau: MSD, PFIZER, BMS, ROCHE, SANOFI, fabrizio conti: None declared, Andrea Doria: None declared

DOI: 10.1136/annrheumdis-2019-eular.4489

\section{AB1290 VITAMIN D AND PAIN SYNDROME IN BREAST CANCER PATIENTS TREATED WITH ADJUVANT LETROZOLE}

Stefania Sciacca, Nadia Melillo, Francesco Paolo Cantatore. University of Foggia Medical Area Departments, Department of Medical and Surgical Science, Foggia, Italy

Background: The third-generation aromatase inhibitors (Als) have shown a favorable overall risk-benefit profile in the upfront adjuvant therapy of postmenopausal estrogen receptor-positive breast cancer. Breast cancer patients treated with long term Als experience arthralgias and musculoskeletal aching often described as bone pain, musculoskeletal disorder, arthralgia.

Objectives: The purpose of this study was to investigate clinical, serological and anamnestic features associated to the joint pain syndrome in non-metastatic breast cancer patients treated with adjuvant Als.

Methods: Between June 2017 and December 2017 patients with early stage estrogen receptor-positive breast carcinomas treated with adjuvant letrozole, attending our Rheumatologic clinic to undergo osteoporosis screening, were included. Pre-existing symptoms and clinical, serological and imaging features were evaluated to assess the type of musculoskeletal disorder. 